\title{
Existence and uniqueness of a common fixed point under a limit contractive condition
}

\author{
Mohammad Imdad', Sunny Chauhan ${ }^{2 *}$ and Muhammad Alamgir Khan ${ }^{3}$
}

\author{
"Correspondence: \\ sun.gkv@gmail.com \\ ${ }^{2}$ Near Nehru Training Centre, H. No. \\ 274, Nai Basti B-14, Bijnor, Uttar \\ Pradesh 246701, India \\ Full list of author information is \\ available at the end of the article
}

\begin{abstract}
In this paper, utilizing the notion of common limit range property for two pairs of self mappings, we prove common fixed point theorems in fuzzy metric spaces under a limit contractive condition, which improve and extend the results of Zhu et al. [Common fixed point theorems of new contractive conditions in fuzzy metric spaces, J. Appl. Math. 2013:145190, 2013]. We also give some examples to demonstrate the validity of the hypotheses of our results. As an application to our main result, we obtain a fixed point theorem for four finite families of self mappings in fuzzy metric space.

MSC: $47 \mathrm{H} 10 ; 54 \mathrm{H} 25$
\end{abstract}

Keywords: fuzzy metric space; weakly compatible mappings; common limit in the range property; fixed point theorems

\section{Introduction}

In 1965, Zadeh [1] initiated the concept of a fuzzy set in his seminal paper. In the last five decades, there have been a tremendous development and growth in fuzzy mathematics. The concept of fuzzy metric space was introduced by Kramosil and Michalek [2] in 1975, which opened an avenue for further development of analysis in such spaces. Thereafter, George and Veeramani [3] modified the concept of fuzzy metric space introduced by Kramosil and Michalek [2] with a view to obtain a Hausdoroff topology, which has very important applications in quantum particle physics, particularly in connection with both string and $\epsilon^{\infty}$ theory (see, [4-6] and references mentioned therein). Fuzzy set theory also has applications in several domains of applied sciences, which include neural network theory, stability theory, mathematical programming, modeling theory, engineering sciences, medical sciences (medical genetics, nervous system), image processing, control theory, communication, etc. Due to richness in applications, this theory progressed by leaps and bounds which also include interesting applications of the theory in diverse areas.

To use this concept in topology and analysis, several researchers have defined fuzzy metric spaces in several ways (e.g. [7-13]). In 2009, Abbas et al. [14] utilized the notions of the property (E.A) (which contains the class of non-compatible self mappings) and the common property (E.A) in fuzzy metric spaces and proved common fixed point theorems. Here, it can be pointed out that the use of the property (E.A) and the common property (E.A) requires the closedness of the underlying subspaces for the existence of common fixed points. Inspired by this observation, Sintunavarat and Kumam [15] coined the idea of 'common limit range property', which never requires the closedness of such subspaces. 
Motivated by the results of Imdad et al. [16], Chauhan [17] extended the notion of common limit range property to two pairs of self mappings in fuzzy metric spaces and proved several results on the existence and uniqueness of common fixed points in fuzzy metric spaces. For the sake of completeness, we refer the readers to [18-38]. Most recently, Zhu et al. [39] proved common fixed point theorems by using the notions of the property (E.A) and the common property (E.A) in George and Veeramani type fuzzy metric spaces (briefly, GV-type) under a new limit contraction condition.

In the present paper, we prove some common fixed point theorems for weakly compatible mappings in fuzzy metric spaces employing the common limit range property. As an application to our main result, we also derive a common fixed point theorem for four finite families of mappings in fuzzy metric spaces using the notion of pairwise commuting families (due to Imdad et al.[40]) and utilize the same to derive common fixed point theorems for six mappings. In the process, many known results (especially the ones contained in Zhu et al. [39]) are enriched and improved. Some related results are also derived besides furnishing illustrative examples.

\section{Preliminaries}

In this section, we present background material required in our subsequent discussion.

Definition 2.1 [41] A binary operation $*:[0,1] \times[0,1] \rightarrow[0,1]$ is a continuous $t$-norm if it satisfies the following conditions:

(1) $*$ is associative and commutative,

(2) $*$ is continuous,

(3) $a * 1=a$ for all $a \in[0,1]$,

(4) $a * b \leq c * d$, whenever $a \leq c$ and $b \leq d$ for all $a, b, c, d \in[0,1]$.

Examples of continuous $t$-norms are $a * b=\min \{a, b\}, a * b=a b$ and $a * b=\max \{a+b-$ $1,0\}$.

Definition 2.2 [3] A 3-tuple $(X, M, *)$ is said to be a fuzzy metric space if $X$ is an arbitrary set, $*$ is a continuous $t$-norm, and $M$ is a fuzzy set on $X^{2} \times(0, \infty)$ satisfying the following conditions: for all $x, y, z \in X, t, s>0$,

$(\mathrm{GV}-1) M(x, y, t)>0$,

$(\mathrm{GV}-2) \quad M(x, y, t)=1$ if and only if $x=y$,

$(\mathrm{GV}-3) \quad M(x, y, t)=M(y, x, t)$,

(GV-4) $M(x, y, t) * M(y, z, s) \leq M(x, z, t+s)$,

$(\mathrm{GV}-5) M(x, y, \cdot):[0, \infty) \rightarrow[0,1]$ is continuous.

In view of (GV-1) and (GV-2), it is worth pointing out that $0<M(x, y, t)<1$ (for all $t>0$ ) provided $x \neq y$ (see [32]). In what follows, fuzzy metric spaces in the sense of George and Veeramani [3] will be referred to as GV-fuzzy metric spaces.

Every metric space is a fuzzy metric space and is called a standard fuzzy metric space (see [3]). Here, we give some new and interesting examples of fuzzy metric spaces due to Gregori et al. [11]. For further details and related examples, one can see [11]. 
Example 2.1 [11] Let $f: X \rightarrow \mathbb{R}^{+}$be a one-one function, and let $g: \mathbb{R}^{+} \rightarrow[0, \infty)$ be an increasing continuous function. For fixed $\alpha, \beta>0$, define $M$ as

$$
M(x, y, t)=\left(\frac{(\min \{f(x), f(y)\})^{\alpha}+g(t)}{(\max \{f(x), f(y)\})^{\alpha}+g(t)}\right)^{\beta},
$$

then $(X, M, *)$ is a fuzzy metric space on $X$, wherein $*$ is the product $t$-norm, i.e., $a * b=a b$.

Example 2.2 [11] Define a function $M$ as

$$
M(x, y, t)=e^{-\frac{d(x, y)}{g(t)}},
$$

then $(X, M, *)$ is a fuzzy metric space on $X$, wherein $*$ is the product $t$-norm and $g: \mathbb{R}^{+} \rightarrow$ $[0, \infty)$ is an increasing continuous function.

Example 2.3 [11] Let $(X, d)$ be a bounded metric space with $d(x, y)<k$ (for all $x, y \in X$ ). Let $g: \mathbb{R}^{+} \rightarrow[k, \infty)$ be an increasing continuous function. Define a function $M$ as

$$
M(x, y, t)=1-\frac{d(x, y)}{g(t)}
$$

then $(X, M, *)$ is a fuzzy metric space on $X$, wherein $*$ is a Lukasiewicz $t$-norm, i.e., $a * b=$ $\max \{a+b-1,0\}$.

Definition 2.3 [32] A sequence $\left\{x_{n}\right\}$ in a GV-fuzzy metric space $(X, M, *)$ is said to be convergent to some $x \in X$ if for all $t>0$, there is some $n_{0} \in \mathbb{N}$ such that

$$
\lim _{n \rightarrow \infty} M\left(x_{n}, x, t\right)=1
$$

for all $n \geq n_{0}$.

Definition 2.4 [42] A pair $(A, S)$ of self mappings of a GV-fuzzy metric space $(X, M, *)$ is said to be compatible if and only if $M\left(A S x_{n}, S A x_{n}, t\right) \rightarrow 1$ for all $t>0$, whenever $\left\{x_{n}\right\}$ is a sequence in $X$ such that $A x_{n}, S x_{n} \rightarrow z$ for some $z \in X$ as $n \rightarrow \infty$.

Definition 2.5 [43] A pair $(A, S)$ of self mappings of a non-empty set $X$ is said to be weakly compatible (or coincidentally commuting) if they commute at their coincidence points, that is, if $A z=S z$ some $z \in X$, then $A S z=S A z$.

Definition 2.6 [14] A pair $(A, S)$ of self mappings of a GV-fuzzy metric space $(X, M, *)$ is said to satisfy the property (E.A) if there exists a sequence $\left\{x_{n}\right\}$ in $X$ such that (for some $z \in X)$

$$
\lim _{n \rightarrow \infty} A x_{n}=\lim _{n \rightarrow \infty} S x_{n}=z
$$

Definition 2.7 [14] Two pairs $(A, S)$ and $(B, T)$ of self mappings of a GV-fuzzy metric space $(X, M, *)$ are said to satisfy the common property (E.A) if there exist two sequences 
$\left\{x_{n}\right\}$ and $\left\{y_{n}\right\}$ in $X$ such that (for some $z \in X$ )

$$
\lim _{n \rightarrow \infty} A x_{n}=\lim _{n \rightarrow \infty} S x_{n}=\lim _{n \rightarrow \infty} B y_{n}=\lim _{n \rightarrow \infty} T y_{n}=z .
$$

Definition 2.8 [15] A pair $(A, S)$ of self mappings of a GV-fuzzy metric space $(X, M, *)$ is said to satisfy the $\left(C L R_{S}\right)$ property with respect to mapping $S$ if there exists a sequence $\left\{x_{n}\right\}$ in $X$ such that

$$
\lim _{n \rightarrow \infty} A x_{n}=\lim _{n \rightarrow \infty} S x_{n}=z
$$

where $z \in S(X)$.

It is evident that a pair $(A, S)$ of self mappings satisfying the property (E.A) along with closedness of the subspace $S(X)$ always enjoys the $\left(C L R_{S}\right)$ property.

Definition 2.9 [17] Two pairs $(A, S)$ and $(B, T)$ of self mappings of a GV-fuzzy metric space $(X, M, *)$ are said to satisfy the $\left(C L R_{S T}\right)$ property with respect to mappings $S$ and $T$ if there exist two sequences $\left\{x_{n}\right\}$ and $\left\{y_{n}\right\}$ in $X$ such that

$$
\lim _{n \rightarrow \infty} A x_{n}=\lim _{n \rightarrow \infty} S x_{n}=\lim _{n \rightarrow \infty} B y_{n}=\lim _{n \rightarrow \infty} T y_{n}=z
$$

where $z \in S(X) \cap T(X)$.

Definition 2.10 [40] Two families of self mappings $\left\{A_{i}\right\}_{i=1}^{m}$ and $\left\{S_{k}\right\}_{k=1}^{n}$ are said to be pairwise commuting if

(1) $A_{i} A_{j}=A_{j} A_{i}$ for all $i, j \in\{1,2, \ldots, m\}$,

(2) $S_{k} S_{l}=S_{l} S_{k}$ for all $k, l \in\{1,2, \ldots, n\}$,

(3) $A_{i} S_{k}=S_{k} A_{i}$ for all $i \in\{1,2, \ldots, m\}$ and $k \in\{1,2, \ldots, n\}$.

\section{Results}

On the lines of Zhu et al. [39], let $(X, M, *)$ be a GV-fuzzy metric space, while let $A, B, S$ and $T$ be self mappings of $X$. For any $x, y \in X$ and $t>0$, we write

$$
\begin{aligned}
\min (x, y, t)= & \min \{M(S x, T y, t), M(S x, A x, t), \\
& M(T y, B y, t), M(S x, B y, t), M(T y, A x, t)\} .
\end{aligned}
$$

(C-1) $0<\underline{\lim }_{n \rightarrow \infty} \min \left(x_{n}, y_{n}, t\right)=L(t)<1$ implies that $\varlimsup_{n \rightarrow \infty} \min \left(A x_{n}, B y_{n}, t\right)>L(t)$ for all $t>0$ and any sequence $\left\{x_{n}\right\},\left\{y_{n}\right\}$ in $X$.

The following lemma will be needed in our main theorem.

Lemma 3.1 Let $A, B, S$ and $T$ be self mappings of a GV-fuzzy metric space $(X, M, *)$ satisfying conditions (C-1) and (3.1). Suppose that

(1) the pair $(A, S)$ satisfies the $\left(C L R_{S}\right)$ property (or the pair $(B, T)$ satisfies the $\left(C L R_{T}\right)$ property),

(2) $A(X) \subset T(X)($ or $B(X) \subset S(X))$,

(3) $T(X)($ or $S(X))$ is a closed subset of $X$, 
(4) $B\left(y_{n}\right)$ converges for every sequence $\left\{y_{n}\right\}$ in $X$, whenever $T\left(y_{n}\right)$ converges (or $A\left(x_{n}\right)$ converges for every sequence $\left\{x_{n}\right\}$ in $X$, whenever $S\left(x_{n}\right)$ converges).

Then the pairs $(A, S)$ and $(B, T)$ satisfy the $\left(C L R_{S T}\right)$ property.

Proof Since the pair $(A, S)$ satisfies the $\left(C L R_{S}\right)$ property with respect to mapping $S$, there exists a sequence $\left\{x_{n}\right\}$ in $X$ such that

$$
\lim _{n \rightarrow \infty} A x_{n}=\lim _{n \rightarrow \infty} S x_{n}=z
$$

where $z \in S(X)$. As $A(X) \subset T(X)$, for every sequence $\left\{x_{n}\right\}$, there exists a sequence $\left\{y_{n}\right\}$ in $X$ such that $A x_{n}=T y_{n}$. Owing to closedness of $T(X)$,

$$
\lim _{n \rightarrow \infty} T y_{n}=\lim _{n \rightarrow \infty} A x_{n}=z
$$

where $z \in S(X) \cap T(X)$. Thus in all, we have $A x_{n} \rightarrow z, S x_{n} \rightarrow z$ and $T y_{n} \rightarrow z$ as $n \rightarrow \infty$. Now, we require to show that $B y_{n} \rightarrow z$ as $n \rightarrow \infty$. By using (3.1) with $x=x_{n}, y=y_{n}$ and any $t>0$, we have

$$
\begin{aligned}
\varliminf_{n \rightarrow \infty} \min \left(x_{n}, y_{n}, t\right)= & \varliminf_{n \rightarrow \infty} \min \left\{M\left(S x_{n}, T y_{n}, t\right), M\left(S x_{n}, A x_{n}, t\right), M\left(T y_{n}, B y_{n}, t\right),\right. \\
& \left.M\left(S x_{n}, B y_{n}, t\right), M\left(T y_{n}, A x_{n}, t\right)\right\} \\
= & \varliminf_{n \rightarrow \infty} M\left(z, B y_{n}, t\right),
\end{aligned}
$$

or

$$
\varliminf_{n \rightarrow \infty} \min \left(x_{n}, y_{n}, t\right)=\varlimsup_{n \rightarrow \infty} M\left(z, B y_{n}, t\right)=\lim _{n \rightarrow \infty} M\left(z, B y_{n}, t\right) .
$$

If $B y_{n} \nrightarrow z$ as $n \rightarrow \infty$, then there is some $t>0$ such that $0<M\left(B y_{n}, z, t\right)<1$ as $n \rightarrow \infty$. Hence, (C-1) implies that

$$
\lim _{n \rightarrow \infty} M\left(B y_{n}, z, t\right)>\varliminf_{n \rightarrow \infty} \min \left(x_{n}, y_{n}, t\right)
$$

which is a contradiction. Hence, $B y_{n} \rightarrow z$, which shows that the pairs $(A, S)$ and $(B, T)$ enjoy the $\left(C L R_{S T}\right)$ property. This completes the proof of lemma.

Remark 3.1 In general, the converse of Lemma 3.1 is not true (see [40, Example 3.5]).

Now, we state and prove our main result for two pairs of weakly compatible mappings satisfying the $\left(C L R_{S T}\right)$ property.

Theorem 3.1 Let $A, B, S$ and $T$ be self mappings of a $G V$-fuzzy metric space $(X, M, *)$ satisfying conditions $(C-1)$ and (3.1). If the pairs $(A, S)$ and $(B, T)$ share the $\left(C L R_{S T}\right)$ property, then $(A, S)$ and $(B, T)$ have a coincidence point each. Moreover, $A, B, S$ and $T$ have a unique common fixed point provided both pairs $(A, S)$ and $(B, T)$ are weakly compatible. 
Proof If the pairs $(A, S)$ and $(B, T)$ satisfy the $\left(C L R_{S T}\right)$ property, then there exist two sequences $\left\{x_{n}\right\}$ and $\left\{y_{n}\right\}$ in $X$ such that

$$
\lim _{n \rightarrow \infty} A x_{n}=\lim _{n \rightarrow \infty} S x_{n}=\lim _{n \rightarrow \infty} B y_{n}=\lim _{n \rightarrow \infty} T y_{n}=z,
$$

where $z \in S(X) \cap T(X)$. Since $z \in S(X)$, there exists a point $u \in X$ such that $S u=z$. We show that $A u=S u$. By using (3.1) with $x=u, y=y_{n}$, we get (for any $t>0$ )

$$
\begin{aligned}
& \varliminf_{n \rightarrow \infty} \min \left(u, y_{n}, t\right) \\
&=\varliminf_{n \rightarrow \infty} \min \left\{M\left(S u, T y_{n}, t\right), M(S u, A u, t), M\left(T y_{n}, B y_{n}, t\right),\right. \\
&\left.M\left(S u, B y_{n}, t\right), M\left(T y_{n}, A u, t\right)\right\} \\
&= M(A u, z, t) \\
&= \lim _{n \rightarrow \infty} M\left(A u, B y_{n}, t\right),
\end{aligned}
$$

or

$$
\varliminf_{n \rightarrow \infty} \min \left(u, y_{n}, t\right)=\varlimsup_{n \rightarrow \infty} M\left(A u, B y_{n}, t\right)=\lim _{n \rightarrow \infty} M(A u, z, t) .
$$

If $A u \neq z$, then there is some $t>0$ such that $0<M(A u, z, t)<1$. Therefore, $(\mathrm{C}-1)$ implies that

$$
\lim _{n \rightarrow \infty} M\left(A u, B y_{n}, t\right)>\varliminf_{n \rightarrow \infty} \min \left(u, y_{n}, t\right),
$$

which is a contradiction. Hence, $A u=S u=z$. Therefore, $u$ is a coincidence point of the pair $(A, S)$.

Also $z \in T(X)$, there exists a point $v \in X$ such that $T v=z$. We assert that $B v=T v$. By using (3.1) with $x=u, y=v$ and $t>0$, we have

$$
\begin{aligned}
\min (u, v, t) & =\min \{M(S u, T v, t), M(S u, A u, t), M(T v, B v, t), M(S u, B v, t), M(T v, A u, t)\} \\
& =M(z, B v, t) .
\end{aligned}
$$

In view of $(C-1)$, we get $M(z, B v, t)=1$, which implies that $B v=z$, and so $B v=T v=z$. Hence, $v$ is a coincidence point of the pair $(B, T)$.

Since the pair $(A, S)$ is weakly compatible, and $A u=S u$, hence, $A z=A S u=S A u=S z$. Now, we show that $z$ is a common fixed point of the pair $(A, S)$. By using (3.1) with $x=z$, $y=v$ and $t>0$, we have

$$
\begin{aligned}
\min (z, v, t) & =\min \{M(S z, T v, t), M(S z, A z, t), M(T v, B v, t), M(S z, B v, t), M(T v, A z, t)\} \\
& =M(A z, z, t) .
\end{aligned}
$$

By (C-1), we obtain $M(A z, z, t)=1$, and so $A z=z=S z$, which shows that $z$ is a common fixed point of the pair $(A, S)$. 
Also the pair $(B, T)$ is weakly compatible, and $B v=T v$, then $B z=B T w=T B w=T z$. By using (3.1) with $x=u, y=z$ and $t>0$, we have

$$
\begin{aligned}
\min (u, z, t) & =\min \{M(S u, T z, t), M(S u, A u, t), M(T z, B z, t), M(S u, B z, t), M(T z, A u, t)\} \\
& =M(z, B z, t) .
\end{aligned}
$$

In view of $(\mathrm{C}-1)$, we get $M(z, B z, t)=1$, which implies that $B z=z$. Therefore, $B z=z=T z$, which shows that $z$ is a common fixed point of the pair $(B, T)$. Hence, $z$ is a common fixed point of both pairs $(A, S)$ and $(B, T)$. Uniqueness of common fixed point is an easy consequence of inequality (3.1). This concludes the proof.

Remark 3.2 Theorem 3.1 improves the corresponding results contained in Zhu et al. [39] as closedness of the underlying subspaces is not required.

Theorem 3.2 Let $A, B, S$ and $T$ be self mappings of a $G V$-fuzzy metric space $(X, M, *)$ satisfying all the hypotheses of Lemma 3.1. Then $A, B, S$ and $T$ have a unique common fixed point provided both pairs $(A, S)$ and $(B, T)$ are weakly compatible.

Proof In view of Lemma 3.1, the pairs $(A, S)$ and $(B, T)$ satisfy the $\left(C L R_{S T}\right)$ property, so that there exist two sequences $\left\{x_{n}\right\}$ and $\left\{y_{n}\right\}$ in $X$ such that

$$
\lim _{n \rightarrow \infty} A x_{n}=\lim _{n \rightarrow \infty} S x_{n}=\lim _{n \rightarrow \infty} T y_{n}=\lim _{n \rightarrow \infty} B y_{n}=z,
$$

where $z \in S(X) \cap T(X)$. The rest of the proof runs on the lines of the proof of Theorem 3.1. This completes the proof.

Let $\phi:[0,1] \rightarrow \mathbb{R}^{+}$be a non-negative and Lebesgue integrable function satisfying

$$
\int_{0}^{\epsilon} \phi(t) d t>0
$$

for each $0<\epsilon \leq 1$. We denote $d=\int_{0}^{1} \phi(t) d t$.

$(\mathrm{C}-2)$ There exists a function $\psi:[0,1] \rightarrow[0,1]$ such that for any $0<s<d, \psi(s)<s$, $\overline{\lim }_{u \rightarrow s} \psi(u)<s$ and for any $x, y \in X, t \in(0, \infty), 0<\min (x, y, t)<1$ implies that

$$
\psi\left(\int_{0}^{M(A x, B y, t)} \phi(s) d s\right) \geq \int_{0}^{\min (x, y, t)} \phi(s) d s .
$$

(C-3) There exists a function $\varphi:[0,1] \rightarrow[0,1]$ such that for any $0<s<d, \varphi(s)>s$, $\underline{\lim }_{t \rightarrow s} \varphi(t)>s$ and for any $x, y \in X, t \in(0, \infty), 0<\min (x, y, t)<1$ implies that

$$
\int_{0}^{M(A x, B y, t)} \phi(s) d s \geq \varphi\left(\int_{0}^{\min (x, y, t)} \phi(s) d s\right),
$$

then (C-1) holds. It can be easily considered from the proof of Theorem 2.2 contained in [39].

By taking $\phi(t)=1$ in conditions $(\mathrm{C}-1)$ and $(\mathrm{C}-2)$, we can deduce the following contractive conditions: 
(C-4) There exists a function $\psi:[0,1] \rightarrow[0,1]$ such that for any $0<s<1, \psi(s)<s$, $\varlimsup_{u \rightarrow s} \psi(u)<s$ and for any $x, y \in X, t \in(0, \infty), 0<\min (x, y, t)<1$ implies that

$$
\psi(M(A x, B y, t)) \geq \min (x, y, t)
$$

(C-5) There exists a function $\varphi:[0,1] \rightarrow[0,1]$ such that for any $0<s<1, \varphi(s)>s$, $\underline{\lim }_{t \rightarrow s} \varphi(t)>s$ and for any $x, y \in X, t \in(0, \infty), 0<\min (x, y, t)<1$ implies that

$$
M(A x, B y, t) \geq \varphi(\min (x, y, t))
$$

Remark 3.3 The conclusion of Lemma 3.1, Theorem 3.1 and Theorem 3.2 remains true in view of conditions (C-2)-(C-5).

Remark 3.4 It may be noted that condition (C-4) is also valid, if one can define a function $\psi:[0,1] \rightarrow[0,1]$ by

(1) $\psi$ is non-increasing for any $0<s<1, \psi(s)<s, \varlimsup_{u \rightarrow s} \psi(u)<s$.

(2) $\psi$ is a upper semi-continuous function such that for any $0<s<1, \psi(s)<s$.

(3) $\psi$ is non-increasing and left-upper semi-continuous such that $\psi(s)<s$ for $0<s<1$.

Remark 3.5 It may be pointed out that condition (C-5) is also valid if one can define a function $\varphi:[0,1] \rightarrow[0,1]$ by

(1) $\varphi$ is a non-decreasing and left continuous function such that for any $0<s<1$, $\varphi(s)>s$.

(2) $\varphi$ is a lower semi-continuous function such that for any $0<s<1, \varphi(s)>s$.

(3) $\varphi(s)=s+\rho(s)$, where $\rho:[0,1] \rightarrow[0,1]$ is a continuous function with for all $0<s<1$, $\rho(s)>0$.

Theorem 3.3 Let $A, B, S$ and $T$ be self mappings of a $G V$-fuzzy metric space $(X, M, *)$. Suppose that there exists an upper semi-continuous function $r:[0,+\infty) \rightarrow[0,+\infty)$ with $r(s)<s$ for every $s>0$ such that

$$
\frac{1}{M(A x, B y, t)}-1 \leq r\left(\frac{1}{\min (x, y, t)}-1\right)
$$

for each $x, y \in X$ and $t>0$. If the pairs $(A, S)$ and $(B, T)$ share the $\left(C L R_{S T}\right)$ property, then $(A, S)$ and $(B, T)$ have a coincidence point each. Moreover, $A, B, S$ and $T$ have a unique common fixed point provided both pairs $(A, S)$ and $(B, T)$ are weakly compatible.

Proof Consider the function $\varphi:[0,1] \rightarrow[0,1]$ as

$$
\varphi(t)= \begin{cases}0, & \text { if } t=0 \\ \frac{1}{1+r\left(\frac{1}{t}-1\right)}, & \text { if } 0<t \leq 1\end{cases}
$$

For every $s>0$, we have

$$
\begin{aligned}
\varliminf_{t \rightarrow s} \varphi(t) & =\frac{1}{1+\overline{\lim }_{t \rightarrow s} r\left(\frac{1}{t}-1\right)} \\
& =\frac{1}{1+r\left(\frac{1}{s}-1\right)}
\end{aligned}
$$




$$
\begin{aligned}
& >\frac{1}{1+\frac{1}{s}-1} \\
& =s .
\end{aligned}
$$

Hence, inequality (3.7) can be deduced by

$$
M(A x, B y, t) \geq \varphi(\min (x, y, t))
$$

for any $x, y \in X, t \in(0,1), 0<\min (x, y, t)<1$, that is, condition $(C-5)$ holds. The rest of the proof can be completed easily, hence, we did not include the details.

Theorem 3.4 Let $A, B, S$ and $T$ be self mappings of a $G V$-fuzzy metric space $(X, M, *)$. Suppose that there exists a strictly decreasing and left continuous function $v:[0,1] \rightarrow[0,1]$ with $\nu(\lambda)=0$ if and only if $\lambda=1$ and a function $k:(0, \infty) \rightarrow(0,1)$ such that

$$
v(M(A x, B y, t)) \leq k(t) \cdot v(\min (x, y, t))
$$

for each $x, y \in X$, where $(x \neq y)$ and $t>0$. If the pairs $(A, S)$ and $(B, T)$ share the $\left(C L R_{S T}\right)$ property, then $(A, S)$ and $(B, T)$ have a coincidence point each. Moreover, $A, B, S$ and $T$ have a unique common fixed point provided both pairs $(A, S)$ and $(B, T)$ are weakly compatible.

Proof Consider a function $\varphi:[0,1] \times[0,+\infty) \rightarrow[0,1]$ as

$$
\varphi(s, t)=v^{-1}(k(t) v(s)) .
$$

Since $v$ is strictly decreasing and left continuous, we have that $v^{-1}$ is strictly decreasing and right continuous, and $\varphi(s, t)$ is increasing in $s$. Then we have

$$
\varliminf_{u \rightarrow s^{-}} \varphi(u, t)=v^{-1}(k(t) v(s))
$$

Also we have that $\varphi(u, t) \geq \varphi(s, t)$ for $u>s$, which shows that

$$
\varliminf_{u \rightarrow s^{+}} \varphi(u, t) \geq v^{-1}(k(t) v(s))
$$

Hence, we get

$$
\varliminf_{u \rightarrow s} \varphi(u, t) \geq \varphi(s, t)=v^{-1}(k(t) v(s))>v^{-1}(v(s))=s .
$$

Therefore, inequality (3.8) can be written as

$$
M(A x, B y, t) \geq \varphi(\min (x, y, t), t) .
$$

If $\left\{x_{n}\right\},\left\{y_{n}\right\}$ in $X$ and $0<\underline{\lim }_{n \rightarrow \infty} \min \left(x_{n}, y_{n}, t\right)=L(t)<1$, then there exists a subsequence $\left\{\min \left(x_{n_{i}}, y_{n_{i}}, t\right)\right\}$ such that $\lim _{i \rightarrow \infty} \min \left(x_{n_{i}}, y_{n_{i}}, t\right)=L$, which shows that

$$
\varliminf_{i \rightarrow \infty}^{\lim _{i}} \varphi\left(\min \left(x_{n_{i}}, y_{n_{i}}, t\right), t\right) \geq \varliminf_{t \rightarrow l}^{\lim _{1}} \varphi(t, t)=\varliminf_{i \rightarrow \infty}^{\lim _{i}} \min \left(x_{n_{i}}, y_{n_{i}}, t\right) .
$$


It follows from $M(A x, B y, t) \geq \varphi(\min (x, y, t), t)$, we get

$$
\begin{aligned}
\varlimsup_{n \rightarrow \infty} M\left(A x_{n}, B y_{n}, t\right) & \geq \varlimsup_{n \rightarrow \infty} \varphi\left(\min \left(x_{n}, y_{n}, t\right), t\right) \\
& \geq \varlimsup_{i \rightarrow \infty} \varphi\left(\min \left(x_{n_{i}}, y_{n_{i}}, t\right), t\right) \\
& =\varlimsup_{n \rightarrow \infty} \varphi\left(\min \left(x_{n_{i}}, y_{n_{i}}, t\right), t\right) \\
& >L(t) .
\end{aligned}
$$

Hence, condition (C-1) holds. The conclusion can be deduced from Theorem 3.1. This completes the proof.

Notice that the conclusion of Lemma 3.1 and Theorem 3.1, Theorem 3.2, Theorem 3.3 and Theorem 3.4 remains if we can replace inequality (3.1) by the following: for any $x, y \in X$ and $t>0$, we define

$$
\min (x, y, t)=\min \{M(S x, T y, t), M(S x, A x, t), M(T y, B y, t)\}
$$

By choosing $A, B, S$ and $T$ suitably, we can deduce corollaries involving two as well as three self mappings. For the sake of naturality, we only derive the following corollary involving a pair of self mappings:

Let $(X, M, *)$ be a GV-fuzzy metric space, let $A$ and $S$ be self mappings of a set $X$. For any $x, y \in X$ and $t>0$, we define

$$
\begin{aligned}
\min (x, y, t)= & \min \{M(S x, S y, t), M(S x, A x, t), \\
& M(S y, A y, t), M(S x, A y, t), M(S y, A x, t)\} .
\end{aligned}
$$

(D-1) $0<\underline{\lim }_{n \rightarrow \infty} \min \left(x_{n}, y_{n}, t\right)=L(t)<1$ implies that $\varlimsup_{n \rightarrow \infty} \min \left(A x_{n}, A y_{n}, t\right)>L(t)$ for all $t>0$ and any sequence $\left\{x_{n}\right\},\left\{y_{n}\right\}$ in $X$.

Corollary 3.1 Let $A$ and $S$ be self mappings of a $G V$-fuzzy metric space $(X, M, *)$ satisfying conditions (D-1) and (3.10). If the pair $(A, S)$ enjoys the $\left(C L R_{S}\right)$ property, then $(A, S)$ has a coincidence point. Moreover, $A$ and $S$ have a unique common fixed point provided the pair $(A, S)$ is weakly compatible.

Theorem 3.5 Let $\left\{A_{i}\right\}_{i=1}^{m},\left\{B_{j}\right\}_{j=1}^{n},\left\{S_{k}\right\}_{k=1}^{p}$ and $\left\{T_{l}\right\}_{l=1}^{q}$ be four finite families of self mappings of a GV-fuzzy metric space $(X, M, *)$ with $A=A_{1} A_{2} \cdots A_{m}, B=B_{1} B_{2} \cdots B_{n}, S=S_{1} S_{2} \cdots S_{p}$ and $T=T_{1} T_{2} \cdots T_{q}$ satisfying one of the conditions (C-1)-(C-5) along with (3.1). Suppose that the pairs $(A, S)$ and $(B, T)$ share the $\left(C L R_{S T}\right)$ property, then $(A, S)$ and $(B, T)$ have a point of coincidence each.

Moreover, $\left\{A_{i}\right\}_{i=1}^{m},\left\{B_{j}\right\}_{j=1}^{n},\left\{S_{k}\right\}_{k=1}^{p}$ and $\left\{T_{l}\right\}_{l=1}^{q}$ have a unique common fixed point if the families $\left(\left\{A_{i}\right\},\left\{S_{k}\right\}\right)$ and $\left(\left\{B_{r}\right\},\left\{T_{h}\right\}\right)$ commute pairwise, wherein $i \in\{1,2, \ldots, m\}, k \in\{1,2, \ldots, p\}$, $j \in\{1,2, \ldots, n\}$ and $l \in\{1,2, \ldots, q\}$.

Proof The proof of this theorem can be completed on the lines of Gopal et al. [25]

Remark 3.6 A result similar to Theorem 3.5 can be outlined in respect of Theorem 3.1. 
Now, we indicate that Theorem 3.5 can be utilized to derive common fixed point theorems for any finite number of mappings. As a sample for five mappings, we can derive the following by setting two families of two members, while the rest two of single members.

Let $(X, M, *)$ be a GV-fuzzy metric space, let $A, B, R, S$ and $T$ be self mappings of a set $X$. For any $x, y \in X$ and $t>0$, we define

$$
\begin{aligned}
\min (x, y, t)= & \min \{M(S R x, T y, t), M(S R x, A x, t), \\
& M(T y, B y, t), M(S R x, B y, t), M(T y, A x, t)\} .
\end{aligned}
$$

Corollary 3.2 Let $A, B, R, S$ and $T$ be self mappings of a $G V$-fuzzy metric space $(X, M, *)$ satisfying one of the conditions (C-1)-(C-5) along with inequality (3.11). Suppose that the pairs $(A, S R)$ and $(B, T)$ share the $\left(C L R_{(S R)(T)}\right)$ property. Then $(A, S R)$ and $(B, T)$ have a coincidence point each. Moreover, $A, B, R, S$ and $T$ have a unique common fixed point provided both pairs $(A, S R)$ and $(B, T)$ commute pairwise, that is, $A S=S A, A R=R A, S R=$ $R S, B T=T B$.

Similarly, we can derive a common fixed point theorem for six mappings by setting two families of two members, while the rest two of single members.

Let $(X, M, *)$ be a GV-fuzzy metric space, let $A, B, H, R, S$ and $T$ be self mappings of a set $X$. For any $x, y \in X$ and $t>0$, we define

$$
\begin{aligned}
\min (x, y, t)= & \min \{M(S R x, T H y, t), M(S R x, A x, t), \\
& M(T H y, B y, t), M(S R x, B y, t), M(T H y, A x, t)\} .
\end{aligned}
$$

Corollary 3.3 Let $A, B, H, R, S$ and $T$ be self mappings of a $G V$-fuzzy metric space $(X, M, *)$ satisfying one of the conditions (C-1)-(C-5) along with inequality (3.12). Suppose that the pairs $(A, S R)$ and $(B, T H)$ share the $\left(C L R_{(S R)(T H)}\right)$ property. Then $(A, S R)$ and $(B, T H)$ have a coincidence point each. Moreover, $A, B, R, S$ and $T$ have a unique common fixed point provided both pairs $(A, S R)$ and $(B, T)$ commute pairwise, that is, $A S=S A, A R=R A, S R=$ $R S, B T=T B, B H=H B$ and $T H=H T$.

By setting $A_{1}=A_{2}=\cdots=A_{m}=A, B_{1}=B_{2}=\cdots=B_{n}=B, S_{1}=S_{2}=\cdots=S_{p}=S$ and $T_{1}=T_{2}=\cdots=T_{q}=T$ in Theorem 3.5, we deduce the following:

Let $(X, M, *)$ be a $\mathrm{GV}$-fuzzy metric space, let $A, B, S$ and $T$ be self mappings of a set $X$. For any $x, y \in X$ and $t>0$, we define

$$
\begin{aligned}
\min (x, y, t)= & \min \left\{M\left(S^{p} x, T^{q} y, t\right), M\left(S^{p} x, A^{m} x, t\right),\right. \\
& \left.M\left(T^{q} y, B^{n} y, t\right), M\left(S^{p} x, B^{n} y, t\right), M\left(T^{q} y, A^{m} x, t\right)\right\},
\end{aligned}
$$

where $m, n, p, q$ are fixed positive integers.

(E-1) $0<\underline{\lim }_{n \rightarrow \infty} \min \left(x_{n}, y_{n}, t\right)=L(t)<1$ implies that $\varlimsup_{n \rightarrow \infty} \min \left(A^{m} x_{n}, B^{n} y_{n}, t\right)>L(t)$ for all $t>0$ and any sequence $\left\{x_{n}\right\},\left\{y_{n}\right\}$ in $X$.

Corollary 3.4 Let $A, B, S$ and $T$ be self mappings of a $G V$-fuzzy metric space $(X, M, *)$ satisfying condition (E-1) with inequality (3.13). Suppose that the pairs $\left(A^{m}, S^{p}\right)$ and $\left(B^{n}, T^{q}\right)$ 
share the $\left(C L R_{S^{p}, T^{q}}\right)$ property. Then $A, B, S$ and $T$ have a unique common fixed point provided $A S=S A$ and $B T=T B$.

Remark 3.7 Corollary 3.4 is a slight but partial generalization of Theorem 3.1 as the commutativity requirements (that is, $A S=S A$ and $B T=T B$ ) in this corollary are relatively stronger as compared to weak compatibility in Theorem 3.1.

Remark 3.8 Results similar to Corollary 3.4 can be derived from Theorem 3.1.

\section{Illustrative examples}

Example 4.1 Let $(X, M, *)$ be a fuzzy metric space, where $X=[3,23)$, with $t$-norm $*$ is defined by $a * b=\min \{a, b\}$ for all $a, b \in[0,1]$ and

$$
M(x, y, t)= \begin{cases}\frac{t}{t+|x-y|}, & \text { if } t>0 \\ 0, & \text { if } t=0,\end{cases}
$$

for all $x, y \in X$. Define the self mappings $A, B, S$ and $T$ by

$$
\begin{aligned}
& A(x)=\left\{\begin{array}{ll}
3, & \text { if } x \in\{3\} \cup(11,23) ; \\
15, & \text { if } x \in(3,11] ;
\end{array} \quad B(x)= \begin{cases}3, & \text { if } x \in\{3\} \cup(11,23) ; \\
6, & \text { if } x \in(3,11] ;\end{cases} \right. \\
& S(x)=\left\{\begin{array}{ll}
3, & \text { if } x=3 ; \\
15, & \text { if } x \in(3,11] ; \\
\frac{x+1}{4}, & \text { if } x \in(11,23) ;
\end{array} \quad T(x)= \begin{cases}3, & \text { if } x=3 ; \\
14, & \text { if } x \in(3,11] ; \\
x-8, & \text { if } x \in(11,23) .\end{cases} \right.
\end{aligned}
$$

Consider the sequences $\left\{x_{n}\right\}=\left\{11+\frac{1}{n}\right\}_{n \in \mathbb{N}},\left\{y_{n}\right\}=\{3\}$ or $\left\{x_{n}\right\}=\{3\},\left\{y_{n}\right\}=\left\{11+\frac{1}{n}\right\}_{n \in \mathbb{N}}$, it is obvious that both pairs $(A, S)$ and $(B, T)$ satisfy the $\left(C L R_{S T}\right)$ property.

$$
\lim _{n \rightarrow \infty} A x_{n}=\lim _{n \rightarrow \infty} S x_{n}=\lim _{n \rightarrow \infty} B y_{n}=\lim _{n \rightarrow \infty} T y_{n}=3 \in S(X) \cap T(X) .
$$

It is noted that $A(X)=\{3,15\} \nsubseteq[3,15)=T(X)$ and $B(X)=\{3,6\} \nsubseteq[3,6) \cup\{15\}=S(X)$. Notice that $S(X)$ and $T(X)$ are not closed subsets of $X$. Thus, all the conditions of Theorem 3.1 are satisfied, and 3 is a unique common fixed point of the pairs $(A, S)$ and $(B, T)$, which also remains a point of coincidence. Also all the involved mappings are even discontinuous at their unique common fixed point 3.

Example 4.2 In the setting of Example 4.1, replace the self mappings $S$ and $T$ by the following, besides retaining the rest:

$$
S(x)=\left\{\begin{array}{ll}
3, & \text { if } x=3 ; \\
6, & \text { if } x \in(3,11] ; \\
\frac{x+1}{4}, & \text { if } x \in(11,23) ;
\end{array} \quad T(x)= \begin{cases}3, & \text { if } x=3 \\
15, & \text { if } x \in(3,11] \\
x-8, & \text { if } x \in(11,23)\end{cases}\right.
$$

Hence, the pairs $(A, S)$ and $(B, T)$ satisfy the $\left(C L R_{S T}\right)$ property. It is noted that $A(X)=$ $\{3,15\} \subset[3,15]=T(X)$ and $B(X)=\{3,6\} \subset[3,6]=S(X)$. The pairs $(A, S)$ and $(B, T)$ commute at 3 , which is also their common coincidence point. Thus, all the conditions of Theorem 3.2 are satisfied, and 3 is a unique common fixed point of the involved mappings $A$, 
$B, S$ and $T$. Here, it may be pointed out that Theorem 3.1 is not applicable to this example, as $S(X)$ and $T(X)$ are closed subsets of $X$.

Our next example shows that if (C-1) does not hold, then the involved mappings may have no common fixed point.

Example 4.3 Let $(X, M, *)$ be a fuzzy metric space, where $X=[0,1]$ with $t$-norm $*$ is defined by $a * b=\min \{a, b\}$ for all $a, b \in[0,1]$, and

$$
M(x, y, t)= \begin{cases}\frac{t}{t+|x-y|}, & \text { if } t>0 \\ 0, & \text { if } t=0\end{cases}
$$

for all $x, y \in X$. Define the self mappings $A=B$ and $S=T$ by

$$
A(x)=\left\{\begin{array}{ll}
\frac{1}{5}, & \text { if } x=0 ; \\
\frac{x}{4}, & \text { if } x \in(0,1) ; \\
0, & \text { if } x=1 ;
\end{array} \quad S(x)= \begin{cases}0, & \text { if } x=0 ; \\
\frac{x}{8}, & \text { if } x \in(0,1) ; \\
\frac{1}{10}, & \text { if } x=1 .\end{cases}\right.
$$

Then

(1) The mappings $A$ and $S$ satisfy the $\left(C L R_{S}\right)$ property for the sequence $x_{n}=\frac{1}{n}$,

$n=1,2, \ldots$, i.e.,

$$
\lim _{n \rightarrow \infty} A x_{n}=\lim _{n \rightarrow \infty} S x_{n}=0 \in\left[0, \frac{1}{8}\right)=S(X) .
$$

(2) The pair $(A, S)$ is weakly compatible.

(3) $A(X)=\left[0, \frac{1}{4}\right) \nsubseteq\left[0, \frac{1}{8}\right)=S(X)$, wherein $A(X)$ and $S(X)$ are not closed subsets of $X$.

(4) (C-1) does not hold. In fact, for $x_{n}=\frac{1}{n}$ and $y_{n}=0, n=1,2, \ldots$, we have

$$
\begin{aligned}
\min \left(x_{n}, y_{n}, t\right) & =\min \left\{\begin{array}{c}
M\left(S x_{n}, T y_{n}, t\right), M\left(S x_{n}, A x_{n}, t\right), M\left(T y_{n}, B y_{n}, t\right), \\
M\left(S x_{n}, B y_{n}, t\right), M\left(T y_{n}, A x_{n}, t\right)
\end{array}\right\} \\
& =\min \left\{\begin{array}{c}
M\left(\frac{1}{8 n}, 0, t\right), M\left(\frac{1}{8 n}, \frac{1}{4 n}, t\right), M\left(0, \frac{1}{5}, t\right), \\
M\left(\frac{1}{8 n}, \frac{1}{5}, t\right), M\left(0, \frac{1}{4 n}, t\right)
\end{array}\right\} \\
& =\min \left\{\frac{t}{t+\frac{1}{8 n}}, \frac{t}{t+\frac{1}{8 n}}, \frac{t}{t+\frac{1}{5}}, \frac{t}{t+\frac{1}{5}-\frac{1}{8 n}}, \frac{t}{t+\frac{1}{4 n}}\right\} \\
& =\frac{t}{t+\frac{1}{5}} \quad(n \rightarrow \infty) .
\end{aligned}
$$

And

$$
\varlimsup_{n \rightarrow \infty} M\left(A x_{n}, B y_{n}, t\right)=\varlimsup_{n \rightarrow \infty} M\left(\frac{1}{4 n}, \frac{1}{5}, t\right)=\varlimsup_{n \rightarrow \infty} \frac{t}{t+\frac{1}{5}-\frac{1}{4 n}}=\frac{t}{t+\frac{1}{5}},
$$

which shows that (C-1) does not hold.

(5) $A$ and $S$ have no common fixed point.

Thus, (C-1) is important for the existence of common fixed point. 


\section{Author details}

'Department of Mathematics, Aligarh Muslim University, Aligarh, 202 002, India. ${ }^{2}$ Near Nehru Training Centre, H. No. 274, Nai Basti B-14, Bijnor, Uttar Pradesh 246701, India. ${ }^{3}$ Department of Natural Resources Engineering and Management, University of Kurdistan, Hewler, Iraq.

\section{Received: 4 June 2013 Accepted: 25 September 2013 Published: 09 Nov 2013}

\section{References}

1. Zadeh, LA: Fuzzy sets. Inf. Control 8, 338-353 (1965) MR0219427 (36 \#2509)

2. Kramosil, I, Michalek, J: Fuzzy metric and statistical metric spaces. Kybernetika 11(5), 336-344 (1975) MR0410633 $(53$ $\# 14381)$

3. George, A, Veeramani, P: On some result in fuzzy metric space. Fuzzy Sets Syst. 64, 395-399 (1994) MR1289545

4. El Naschie, MS: On the uncertainty of Cantorian geometry and two-slit experiment. Chaos Solitons Fractals 9(3), 517-529 (1998) MR1625647

5. El Naschie, MS: A review of E-infinity theory and the mass spectrum of high energy particle physics. Chaos Solitons Fractals 19, 209-236 (2004)

6. El Naschie, MS: A review of applications and results of E-infinity theory. Int. J. Nonlinear Sci. Numer. Simul. 8, 11-20 (2007)

7. Cho, YJ: Fixed points in fuzzy metric spaces. J. Fuzzy Math. 5(4), 949-962 (1997) MR1488041 (98h:54011)

8. Cho, YJ, Pathak, HK, Kang, SM, Jung, JS: Common fixed points of compatible maps of type ( $\beta$ ) on fuzzy metric spaces. Fuzzy Sets Syst. 93(1), 99-111 (1998) MR1600404

9. Cho, YJ, Sedghi, S, Shobe, N: Generalized fixed point theorems for compatible mappings with some types in fuzzy metric spaces. Chaos Solitons Fractals 39(5), 2233-2244 (2009) MR2519428 (2010e:54002)

10. Grabiec, M: Fixed points in fuzzy metric spaces. Fuzzy Sets Syst. 27(3), 385-389 (1988) MR0956385 (89:54007)

11. Gregori, V, Morillas, S, Sapena, A: Examples of fuzzy metrics and applications. Fuzzy Sets Syst. 170, 95-111 (2011)

12. Subrahmanyam, PV: A common fixed point theorem in fuzzy metric spaces. Inf. Sci. 83(3-4), 109-112 (1995) MR1323308

13. Vasuki, R: Common fixed points for R-weakly commuting maps in fuzzy metric spaces. Indian J. Pure Appl. Math. 30(4), 419-423 (1999) MR1695690

14. Abbas, M, Altun, I, Gopal, D: Common fixed point theorems for non compatible mappings in fuzzy metric spaces Bull. Math. Anal. Appl. 1(2), 47-56 (2009) MR2403120

15. Sintunavarat, W, Kumam, P: Common fixed point theorems for a pair of weakly compatible mappings in fuzzy metric spaces. J. Appl. Math. 2011, Article ID 637958 (2011) MR2822403

16. Imdad, M, Pant, BD, Chauhan, S: Fixed point theorems in Menger spaces using the (CLRST) property and applications. J. Nonlinear Anal. Optim. 3(2), 225-237 (2012)

17. Chauhan, S: Fixed points of weakly compatible mappings in fuzzy metric spaces satisfying common limit in the range property. Indian J. Math. 54(3), 375-397 (2012)

18. Beg, I, Sedghi, S, Shobe, N: Fixed point theorems in fuzzy metric spaces. Int. J. Anal. 2013, Article ID 934145 (2013)

19. Chauhan, S, Bhatnagar, S, Radenović, S: Common fixed point theorems for weakly compatible mappings in fuzzy metric spaces. Matematiche 68(1), 87-98 (2013)

20. Chauhan, S, Dhiman, $\mathrm{N}$ : Common fixed point theorems on fuzzy metric spaces using implicit relation. Math. Sci. Lett. 1(2), 89-96 (2012)

21. Chauhan, S, Khan, MA, Kumar, S: Unified fixed point theorems in fuzzy metric spaces via common limit range property. J. Inequal. Appl. 2013, 182 (2013)

22. Chauhan, S, Khan, MA, Sintunavarat, W: Common fixed point theorems in fuzzy metric spaces satisfying $\phi$-contractive condition with common limit range property. Abstr. Appl. Anal. 2013, Article ID 735217 (2013)

23. Chauhan, S, Kumar, S: Coincidence and fixed points in fuzzy metric spaces using common property (E.A). Kochi J. Math. 8, 135-154 (2013)

24. Chauhan, S, Pant, BD: Common fixed point theorems in fuzzy metric spaces. Bull. Allahabad Math. Soc. 27(1), 27-43 (2012)

25. Gopal, D, Imdad, M, Vetro, C: Impact of common property (E.A.) on fixed point theorems in fuzzy metric spaces. Fixed Point Theory Appl. 2011, Article ID 297360 (2011) MR2784507

26. Imdad, M, Ali, J: Some common fixed point theorems in fuzzy metric spaces. Math. Commun. 11(2), 153-163 (2006) MR2298646

27. Imdad, M, Ali, J: A general fixed point theorem in fuzzy metric spaces via an implicit function. J. Appl. Math. Inform. 26(3-4), 591-603 (2008)

28. Imdad, M, Ali, J, Hasan, M: Common fixed point theorems in fuzzy metric spaces employing common property (E.A.). Math. Comput. Model. 55(3-4), 770-778 (2012)

29. Kumar, S, Chauhan, S: Common fixed point theorems using implicit relation and property (E.A) in fuzzy metric spaces. Ann. Fuzzy Math. Inf. 5(1), 107-114 (2013)

30. Kumar, S, Fisher, B: A common fixed point theorem in fuzzy metric space using property (E.A.) and implicit relation. Thai J. Math. 8(3), 439-446 (2010) MR2763666 (2011m:54045)

31. Kutukcu, S, Turkoglu, D, Yildiz, C: Common fixed points of compatible maps of type ( $\beta$ ) on fuzzy metric spaces. Commun. Korean Math. Soc. 21(1), 89-100 (2006) MR2199304 (2006j:54053)

32. Miheţ, D: Fixed point theorems in fuzzy metric spaces using property E.A. Nonlinear Anal. 73(7), 2184-2188 (2010) MR2674194

33. Murthy, PP, Kumar, S, Tas, K: Common fixed points of self maps satisfying an integral type contractive condition in fuzzy metric spaces. Math. Commun. 15(2), 521-537 (2010) MR2814310 (2012d:54046)

34. Pant, BD, Chauhan, S: Common fixed point theorems for two pairs of weakly compatible mappings in Menger spaces and fuzzy metric spaces. Sci. Stud. Res. Ser. Math. Inf. 21(2), 81-96 (2011)

35. Pant, V, Pant, RP: Fixed points in fuzzy metric space for noncompatible maps. Soochow J. Math. 33(4), 647-655 (2007) MR2404591 
36. Sedghi, S, Turkoglu, D, Shobe, N: Common fixed point of compatible maps of type $(\gamma)$ on complete fuzzy metric spaces. Commun. Korean Math. Soc. 24(4), 581-594 (2009) MR2568992

37. Sintunavarat, W, Kumam, P: Common fixed points for R-weakly commuting in fuzzy metric spaces. Ann. Univ. Ferrara, Sez. 7: Sci. Mat. 58(2), 389-406 (2012) MR2996467

38. Sintunavarat, W, Kumam, P: Fixed point theorems for a generalized intuitionistic fuzzy contraction in intuitionistic fuzzy metric spaces. Thai J. Math. 10(1), 123-135 (2012) MR2949144

39. Zhu, J, Wang, Y, Kang, SM: Common fixed point theorems of new contractive conditions in fuzzy metric spaces. J. Appl. Math. 2013, Article ID 145190 (2013)

40. Imdad, M, Ali, J, Tanveer, M: Coincidence and common fixed point theorems for nonlinear contractions in Menger PM spaces. Chaos Solitons Fractals 42(5), 3121-3129 (2009) MR2562820 (2010j:54064)

41. Schweizer, B, Sklar, A: Probabilistic Metric Spaces. North-Holland Series in Probability and Applied Mathematics. North-Holland, New York (1983). ISBN:0-444-00666-4 MR0790314

42. Mishra, SN, Sharma, N, Singh, SL: Common fixed points of maps on fuzzy metric spaces. Int. J. Math. Math. Sci. 17(2), 253-258 (1994) MR1261071

43. Jungck, G, Rhoades, BE: Fixed points for set valued functions without continuity. Indian J. Pure Appl. Math. 29(3), 227-238 (1998) MR1617919

10.1186/1029-242X-2013-519

Cite this article as: Imdad et al.: Existence and uniqueness of a common fixed point under a limit contractive condition. Journal of Inequalities and Applications 2013, 2013:519

\section{Submit your manuscript to a SpringerOpen ${ }^{\circ}$ journal and benefit from:}

- Convenient online submission

Rigorous peer review

- Immediate publication on acceptance

- Open access: articles freely available online

- High visibility within the field

- Retaining the copyright to your article 